\title{
CONSUMO DE ÁLCOOL E A INFLUÊNCIA DO EXERCÍCIO FÍSICO NA ATIVIDADE ENZIMÁTICA DE RATOS WISTAR
}

\author{
ALCOHOL CONSUMPTION AND THE INFLUENCE OF PHYSICAL EXERCISE ON ENZYME \\ ACTIVITY OF WISTAR RATS
}

\author{
CONSUMO DE ALCOHOL Y LA INFLUENCIA DEL EJERCICIO FÍSICO EN LA ACTIVIDAD \\ DE LA ENZIMA DE RATAS WISTAR
}

Thamires Righi'
(Graduanda em Bioquímica)
Camilo Amaro de Carvalho'
(Farmacêutico)
Lucas Mota Ribeiro' $^{1}$
(Graduando em Medicina)
Daise Nunes Queiroz da Cunha'
(Médica Veterinária)
Ana Carolina Silva Paiva' ${ }^{1}$
(Graduanda em Bioquímica)
Antônio José Natali'
(Educador Físico)
Eveline Torres Pereira'1
(Educador Físico)
Luciana Moreira Lima' ${ }^{1}$
(Farmacêutica-Bioquímica)
1. Universidade Federal de Viçosa,
MG, Brasil.

\section{Correspondência:}

Luciana Moreira Lima, Departamento de Medicina e Enfermagem, Universidade Federal de Viçosa. Av. PH Rolfs, s/n, Centro - Viçosa, Minas Gerais. 36570-000. luciana.lima@ufv.br

\section{RESUMO}

Introdução: Biomarcadores vem sendo utilizados para monitorar o uso do álcool e, atualmente, os mais sensíveis e específicos são enzimas hepáticas, por exemplo, gama glutamiltransferase (GGT), alanina aminotransferase (ALT), aspartato aminotransferase (AST) e fosfatase alcalina (ALP). Objetivo: Verificar, a partir da experimentação animal, as alterações provocadas pelo uso de álcool e pela prática de atividade física nas enzimas hepáticas e pancreáticas. Métodos: Vinte e quatro ratos da linhagem Wistar foram distribuídos aleatoriamente em grupos experimentais, alojados em gaiolas com ambiente controlado, divididos de acordo com os tratamentos recebidos. No tratamento inicial, foi administrado álcool aos grupos álcool sedentário (AS) e álcool exercitado (AE) e, ao final da quarta semana, iniciou-se o programa de treinamento físico em esteira com os grupos AE e controle exercitado (CE). A coleta de sangue foi realizada por punção cardíaca ao final de cada experimento. Na análise estatística, utilizou-se teste de análise de variância (ANOVA) seguido de teste de Tukey e teste de Kruskal-Wallis. Resultados: O grupo AS apresentou valores significativamente mais elevados de ALT e ALP quando comparado aos grupos CE e AE, respectivamente. Não foram observadas diferenças significativas entre os quatro grupos estudados para os parâmetros AST, GGT e amilase. Conclusão: A associação entre consumo de álcool e sedentarismo aumentou a liberação das enzimas ALT e ALP em ratos Wistar; a prática de exercício físico aeróbico após abstinência alcoólica evitou o aumento da liberação de ALP no plasma desses animais.

Descritores: alcoolismo/enzimologia, atividade motora, ratos Wistar.

\section{ABSTRACT}

Introduction: Biomarkers have been used to monitor the use of alcohol and currently the most sensitive and specific are the liver enzymes, for example, gamma-glutamyl transferase (GGT), alanine aminotransferase (ALT), aspartate aminotransferase (AST) and alkaline phosphatase (ALP). Objective: To determine through animal experiments the changes caused by alcohol and the physical activity in liver and pancreatic enzymes. Methods: Twenty-four Wistar rats were randomly assigned into experimental groups, housed in cages with controlled environment, divided according to the treatment received. In the initial treatment, alcohol was administered to sedentary alcohol (SA) and exercised alcohol (EA) groups and at the end of the fourth week the program of physical training on a treadmill began with the AE and control exercised (CE) groups. Blood collection was performed by cardiac puncture at the end of each experiment. For the statistical analysis we used analysis of variance (ANOVA) followed by Tukey test and Kruskal-Wallis test. Results: The AS group had significantly higher values of ALT and ALP when compared to CE and EA groups, respectively. No significant differences were observed between the four groups for the parameters AST, GGT and amylase. Conclusion: The association between alcohol consumption and physical inactivity increased the release of the enzymes ALT and ALP in Wistar rats; the practice of aerobic exercise after alcohol withdrawal prevented the increased release of ALP in the plasma of these animals.

Keywords: alcoholism/enzymology, motor activity, rats, Wistar.

\section{RESUMEN}

Introducción: Los biomarcadores se han utilizado para controlar el consumo de alcohol y en la actualidad las enzimas hepáticas son las más sensibles y especificas, por ejemplo, gamma glutamil transferasa (GGT), alanina aminotransferasa (ALT), aspartato aminotransferasa (AST) y fosfatasa alcalina (ALP). Objetivo: Determinar, a partir de experimentos con animales, los cambios provocados por el alcohol y la actividad física en las enzimas hepáticas y pancreáticas. Métodos: Veinticuatro ratones Wistar fueron asignados al azar a los grupos experimentales, fueron alojados en jaulas con ambiente controlado, divididos de acuerdo a los tratamientos recibidos. En el tratamiento inicial, el alcohol se administró a los grupos alcohol sedentario (AS) y alcohol ejercicio (AE) y el final de la cuarta semana, se inició el programa de entrenamiento físico en una caminadora con los grupos AE y el control ejercitado (CE). La recogida de sangre se realizó mediante punción cardiaca al final de cada experimento. En el análisis estadístico se utilizó el análisis de varianza (ANOVA), seguido por la prueba de Tukey y la prueba de Kruskal-Wallis. 
Resultados: El grupo AS tuvo valores significativamente más elevados de ALT y ALP en comparación con los grupos CEY AE, respectivamente. No se observaron diferencias significativas entre los cuatro grupos para los parámetros de AST, GGT y amilasa. Conclusión: La asociación entre el consumo de alcohol y la falta de actividad física aumenta la liberación de las enzimas ALT y ALP en ratones Wistar; la práctica de ejercicio aeróbico después de la retirada del alcohol impidió el aumento de la liberación de ALP en el plasma de estos animales.

Descriptores: alcoholismo/enzimología, actividad motora, ratas Wistar.

\section{INTRODUÇÃO}

O consumo abusivo de álcool é reconhecido como um importante problema de saúde pública em todo o mundo, trazendo sérios problemas aos usuários, sendo que o usuário crônico sofre uma série de alterações orgânicas e fisiológicas ${ }^{1,2}$

Da primeira dose às outras subsequentes, até estabelecida a dependência, ocorre transformações e adaptações neurológicas, comportamentais e fisiológicas ${ }^{3}$. Guimarães et al. ${ }^{4}$ relataram que o abuso/ dependência de álcool estão associados a múltiplas consequências adversas para a saúde.

Doenças pulmonares ${ }^{5}$, cardíacas e cerebrovasculares, eventos fatais e transtornos psiquiátricos ${ }^{6}$, traumas $^{7}$, várias neoplasias ${ }^{8}$, doenças sexualmente transmissíveis ${ }^{9}$ e o desenvolvimento de doenças no fígado decorrente da ingestão alcoólica são as principais alterações fisiológicas descritas devido ao abuso de álcool ${ }^{10}$. Em humanos, assim como em animais, as doenças hepáticas associadas podem abranger quadros como esteatose, cirrose e carcinoma hepatocelular ${ }^{11,12}$.

Traumas hepáticos são acompanhados por alterações nos níveis de enzimas, entre estas, a gama glutamiltransferase (GGT), a alanina aminotransferase (ALT), a aspartato aminotransferase (AST) e a fosfatase alcalina (ALP) ${ }^{11}$. Quanto mais elevado os níveis das enzimas hepáticas, maior o risco de síndromes metabólicas ${ }^{13}$.

Devido à alta incidência de recaída durante o tratamento, principalmente na fase de abstinência, investe-se no desenvolvimento de novos modelos de tratamento além do farmacológico em todo o mundo ${ }^{14}$. Dentre esses modelos sugere-se a prática da atividade física (AF) como coadjuvante à manutenção da saúde, pois esta pode resultar em uma melhora na qualidade de vida do dependente ${ }^{15}$. Exercícios aeróbicos, como corrida, apresentam muitos benefícios para saúde, como melhora nas funções cognitivas, transtornos de humor e sono ${ }^{16}$.

Tem sido relatado em estudo com modelos animais que a atividade física de intensidade moderada aumenta as defesas antioxidantes das células ${ }^{17}$ e promove o controle da hipertensão arterial ${ }^{5}$ e das funções neurológicas ${ }^{18}$. De uma forma geral, induz uma melhora no funcionamento do organismo e das funções corporais prejudicadas, como o metabolismo hepático e as funções cognitivas ${ }^{15,16}$.

O exercício pode aumentar a atividade das enzimas hepáticas envolvidas no metabolismo do álcool ${ }^{16}$ e, tanto o exercício agudo como crônico, aumentam a metabolização do álcool'19.

Segundo Xavier et al. ${ }^{5}$, embora tenha sido demonstrado a proteção induzida pelo exercício físico moderado em animais submetidos concomitantemente à dieta alcoólica, os efeitos do exercício físico sobre a reparação de lesões alcoólica já instaladas foram pouco estudadas.

Acredita-se que os danos causados pelo álcool podem ser revertidos significativamente pelo exercício físico devido a sua proteção contra os danos oxidativos induzidos pelo álcool ${ }^{20,21}$.

O objetivo deste estudo foi verificar, a partir da experimentação animal, as alterações provocadas pelo uso de álcool e pela prática de atividade física nas enzimas hepáticas e pancreáticas de ratos Wistar.

\section{MATERIAL E MÉTODOS}

O protocolo (número 42/2013 (data de aprovação: 28/08/2013) do estudo recebeu parecer favorável da Comissão de Ética no Uso de Animais (CEUA) da Universidade Federal de Viçosa (UFV), Viçosa, Minas Gerais, Brasil, e todos os procedimentos experimentais foram realizados de acordo com os princípios éticos na experimentação animal da Sociedade Brasileira de Ciência em Animais de Laboratório (COBEA).

Foram utilizados 24 ratos, com 90 dias de idade, da linhagem Wistar, provenientes do Biotério Central do Centro de Ciências Biológicas e da Saúde da UFV. Os animais foram distribuídos aleatoriamente em grupos experimentais:

1. Grupo controle (sem álcool) e sedentário (CS; $n=6)$ : animais que não receberam álcool e não foram exercitados.

2. Grupo controle (sem álcool) exercitado (CE; $n=6)$ : animais que não receberam álcool, mas foram exercitados.

3. Grupo alcoólico e exercitado (AE; $n=6)$ : animais que receberam álcool e que foram exercitados.

4. Grupo alcoólico sedentário (AS; n=6): animais que receberam álcool, mas não foram exercitados.

Os animais dos grupos experimentais foram alojados em gaiolas, divididos de acordo com os tratamentos recebidos; em ambiente com temperatura média controlada e no ciclo claro-escuro de 12 horas (7:00 às 19:00 h). Os animais receberam água e ração ad libitum.

No tratamento inicial, foi administrado álcool $(4 \mathrm{~g} / \mathrm{Kg})$ aos animais do grupo AE e AS através da gavage, mantido por um período de 4 semanas. A concentração inicial de etanol foi de 5\% (v/v), aumentada de 5\% (v/v), a cada dois dias, até atingir a concentração final de 20\% (v/v). Essa concentração final foi mantida até completar a quarta semana de tratamento.

Os animais foram pesados a cada dois dias, sendo o volume de álcool reajustado para a manutenção da proporção da solução em 4g/Kg.

Ao final da quarta semana iniciou-se o programa de treinamento físico com os grupos AE e CE. Os animais do grupo AE permaneceram por um período de $24 \mathrm{~h}$ sem administração de álcool e seguiu-se então a adaptação para a corrida na esteira com os dois grupos.

Durante a adaptação os grupos exercitados caminharam a 5m/ mim por $10 \mathrm{~min} /$ dia durante três dias. Após a adaptação foi realizado o teste de exaustão para determinar a velocidade média máxima de corrida (VMC). Os animais iniciaram a uma velocidade de $5 \mathrm{~m} / \mathrm{min}$, com incrementos de $3 \mathrm{~m} / \mathrm{min}$ a cada três minutos até a fadiga de cada animal dos grupos experimentais. O momento da fadiga foi definido e o teste interrompido quando os animais não mantiveram a corrida de acordo com a velocidade da esteira.

O exercício foi realizado cinco dias por semana, por duas semanas consecutivas. No primeiro dia os animais correram por 30 min, sendo essa velocidade aumentada de $10 \mathrm{~min} /$ dia até atingir a velocidade final de 60 min, a qual foi mantida até o final do tratamento. A intensidade utilizada foi de $65 \%$ da VMC, correspondendo a uma atividade física de caráter moderado.

A coleta de sangue foi realizada ao final de todo experimento, $24 \mathrm{~h}$ após o último dia de treinamento físico. Os animais sofreram eutanásia 
por anestesia com isoflurano e retirada total do sangue por punção cardíaca. Os procedimentos experimentais tiveram início após a constatação da perda de todos os reflexos inclusive dos membros posteriores. O sangue coletado foi alocado em tubos sem anticoagulante.

As amostras de sangue obtidas foram rapidamente centrifugadas a 2500rpm por 10 minutos para separação do soro. As atividades enzimáticas plasmáticas da AST, ALT, ALP, amilase e GGT foram determinadas por métodos cinéticos específicos utilizando-se conjuntos diagnósticos comerciais BioSystems ${ }^{\circledR}$ (BioSystems, Curitiba, Paraná, Brasil). A dosagem de GGT também foi realizada utilizando-se o conjunto diagnóstico comercial Labtest ${ }^{\circledR}$ (Labtest, Lagoa Santa, Minas Gerais, Brasil) com o intuito de comparar a concordância entre as duas determinações. Todas as dosagens foram realizadas utilizando-se o analisador BS-200 BioSystems ${ }^{\circledR}$ (BioSystems, Curitiba, Paraná, Brasil) em sistema completamente automatizado. Foram utilizados calibradores e soros-controle comerciais (BioSystems, Curitiba, Paraná, Brasil) para verificar o desempenho dos ensaios.

Na análise estatística, inicialmente todos os dados foram submetidos ao teste de Komolgorov-Smirnov para verificar a pressuposição de normalidade em todas as variáveis. Em seguida o teste de análise de variância (ANOVA) seguido de teste de Tukey foi utilizado para todos os parâmetros, uma vez que apresentaram distribuição normal e homocedasticidade, exceto para o parâmetro GGT, analisado pelo teste de Kruskal-Wallis, uma vez que não apresentou distribuição normal. O nível de significância adotado foi de 0,05. Os testes de correlação de Pearson e Spearman foram utilizados para verificar as correlações lineares entre as variáveis estudadas. Os programas Sigma Stat versão 1.0 (San Jose, California, United States of America) e Graphpad Prism versão 3.0 (San Diego, California, United States of America) foram utilizados para realizar as análises e plotar os gráficos, respectivamente.

Em relação a experimentação animal, o tamanho mínimo da amostra foi definida de acordo com uma variância estimada previamente descritos na literatura ${ }^{10}$. A fórmula proposta por Callegari-Jacques e Cochran foi utilizado para o cálculo. Foi possível verificar diferenças estatísticas com de 5\% de significância, com um número mínimo de cinco animais de cada grupo.

\section{RESULTADOS}

A Tabela 1 apresenta as comparações realizadas entre todos os grupos experimentais.

A Tabela 2 apresenta os resultados obtidos para os parâmetros bioquímicos estudados. O grupo AS apresentou valores significativamente mais elevados de ALT e ALP quando comparado aos grupos CE e $A E$, respectivamente. Não foram observadas diferenças significativas entre os quatro grupos estudados para os parâmetros AST e amilase.

As Figuras 1 e 2 apresentam a distribuição dos valores de ALT e ALP nos grupos estudados, respectivamente. Foram observadas correlações positivas e significativas entre as variáveis ALT e AST $(r=0,45$; $p=0,026)$ e entre as variáveis $G G T-1$ e $G G T-2(r=0,898 ; p<0,001)$, quando comparadas de forma univariada. Entre as demais variáveis não foram observadas correlações estatisticamente significativas.

Tabela 1. Efeito das comparações experimentais.

\begin{tabular}{c|c}
\hline Tratamento & Objetivo \\
\hline AS $\times$ CS & Efeito do uso do álcool em animais sedentários \\
\hline AE $\times$ CE & $\begin{array}{r}\text { Efeito da combinação álcool e atividade física comparada ao } \\
\text { efeito individual da atividade física }\end{array}$ \\
\hline AS $\times$ AE & $\begin{array}{r}\text { Efeito da combinação álcool e atividade física comparado ao } \\
\text { efeito individual do álcool }\end{array}$ \\
\hline CS $\times$ CE & Efeito da atividade física nos animais \\
\hline AS $\times$ CE & $\begin{array}{r}\text { Comparação do efeito individual do álcool em animais } \\
\text { sedentários e o efeito individual da atividade física }\end{array}$ \\
\hline AE $\times$ CS & $\begin{array}{r}\text { Comparação entre o efeito combinado do álcool e exercício } \\
\text { com os animais sedentários }\end{array}$ \\
\hline
\end{tabular}

Tabela 2. Parâmetros bioquímicos da amostra estudada.

\begin{tabular}{c|c|c|c|c|c}
\hline \multirow{2}{*}{ Parâmetros } & \multicolumn{4}{|c|}{ Grupos (n=6) } & \multirow{2}{*}{ p } \\
\cline { 2 - 5 } & CS & CE & AE & AS & \\
\hline AST $(U / L)$ & $170 \pm 59$ & $139 \pm 18$ & $146 \pm 29$ & $168 \pm 24$ & 0,357 \\
\hline ALT $(U / L)$ & $77 \pm 9$ & $71 \pm 10$ & $74 \pm 9$ & $87 \pm 6^{\mathrm{a}}$ & $0,029^{\mathrm{a}}$ \\
\hline ALP $(\mathrm{U} / \mathrm{L})$ & $127 \pm 26$ & $200 \pm 65$ & $165 \pm 37$ & $222 \pm 79^{\mathrm{b}}$ & $0,035^{\mathrm{b}}$ \\
\hline GGT $-1(\mathrm{U} / \mathrm{L})^{*}$ & $1,5(1,0-2,0)$ & $1,0(1,0-2,0)$ & $1,0(1,0-2,0)$ & $1,0(1,0-2,0)$ & 0,828 \\
\hline GGT $-2(\mathrm{U} / \mathrm{L})$ & $2,23 \pm 0,81$ & $1,63 \pm 0,61$ & $1,57 \pm 0,60$ & $1,60 \pm 0,57$ & 0,270 \\
\hline Amilase $(\mathrm{U} / \mathrm{L})$ & $582 \pm 67$ & $575 \pm 58$ & $610 \pm 76$ & $634 \pm 61$ & 0,405 \\
\hline
\end{tabular}

CS=controle (sem álcool) sedentário; $\mathrm{CE}=$ controle (sem álcool) exercitado; $\mathrm{AE}=$ =alcoólico exercitado; $\mathrm{AS}=$ =alcoólico sedentário; AST=aspartato aminotransferase; $\mathrm{ALT}=$ alanina aminotransferase; $\mathrm{ALP}=$ fosfatase alcalina; GGT-1=gama glutamiltransferase; GGT-2=gama glutamiltransferase. Dados apresentados como média e desvio padrão (* dados apresentados como mediana e diferença interquartil. As diferenças significativas estão representadas pelas letras: a vs Grupo CE; b vs Grupo CS e AE (ANOVA seguida de Teste de Tukey).

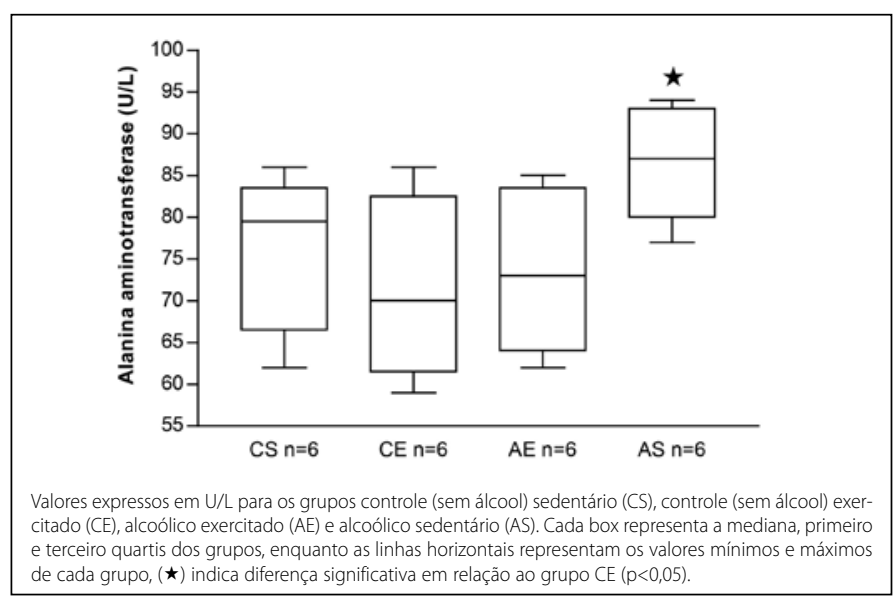

Figura 1. Distribuição dos valores da alanina aminotransferase nos grupos estudados.

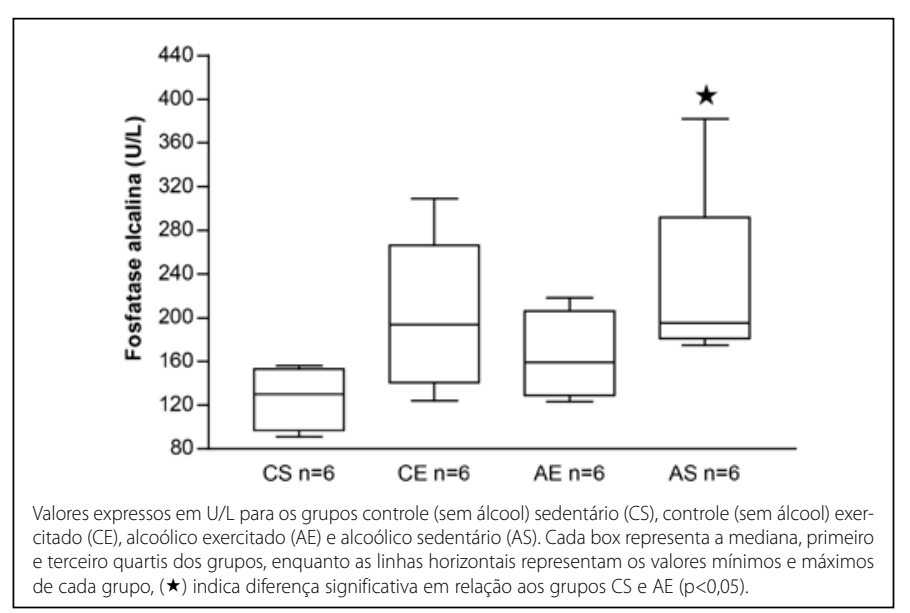

Figura 2. Distribuição dos valores de fosfatase alcalina nos grupos estudados.

\section{DISCUSSÃO}

O principal resultado foi que o consumo de álcool durante quatro semanas promoveu uma maior liberação das enzimas ALT e ALP nos animais sedentários. Além disso, a prática de corrida aeróbica em esteira por duas semanas evitou uma maior liberação plasmática de ALP nos ratos que consumiram álcool.

Observou-se que grupo de animais que recebeu etanol e permaneceu sedentário (AS) no protocolo experimental apresentou níveis mais elevados das atividades plasmáticas das enzimas ALT e ALP, quando comparado com os demais grupos estudados. Esses dados permitem inferir que a associação do sedentarismo e consumo de álcool pode ter promovido uma maior liberação destas enzimas para o plasma.

Não há na literatura dados que demonstrem se existe uma relação 
direta entre a prática de atividade física e a redução das aminotransferases em etilistas, sendo que os níveis dessas enzimas variam de acordo com múltiplos fatores, como o índice de massa corporal e a idade ${ }^{22-24}$.

Diversos autores $3,6,7,10,11$ tentam demonstrar os mecanismos envolvidos na injuria hepática causada pelo álcool que corroboram para a elevação plasmática das aminotransferases. O etanol é oxidado no fígado até a forma de acetaldeído, que em excesso compromete a função de organelas dos hepatócitos, em especial as mitocôndrias. A alteração desse equilíbrio entre meio interno e externo culmina com o processo de morte celular e liberação das enzimas intracelulares para o plasma. Outro mecanismo proposto é que a ingestão crônica de etanol conduz a uma diminuição de substâncias antioxidantes produzidas no próprio fígado, incluindo a superóxido dismutase, catalase e enzimas da família da glutationa, levando ao aumento de radicais livres que contribuem com a lesão hepática ${ }^{25}$.

Os dados do presente estudo mostram que a atividade da enzima ALP foi significativamente mais elevada no grupo AS quando comparado com o grupo CS e AE. Acredita-se que haja relação entre a prática de exercícios aeróbicos e a redução da concentração plasmática de álcool em ratos, o que resulta em menor agressão ao tecido hepático.

Os estudos realizados por Ardies et al. ${ }^{19}$ corroboram com essa teoria indicando que tanto o exercício agudo quanto treinamento físico regular podem colaborar para o reduzir as taxas de etanol no sangue pelo aumento de sua excreção urinária. A longo prazo, a atividade física parece aumentar o metabolismo de etanol pelo sistema microssomal hepático, sem alterar a atividade da enzima álcool desidrogenase hepática, grande responsável pela formação de acetaldeido ${ }^{19,26}$.

Outros autores consideram que a prática da atividade física regular tem como propriedade a otimização da capacidade antioxidante em diversos tecidos do organismo, incluindo o fígado ${ }^{27,28}$. Estudos anteriores demonstraram que o treino de resistência pode melhorar os níveis previamente reduzidos de enzimas antioxidantes hepáticas em ratos tratados com álcool ${ }^{29}$.

Foi observado que a comparação dos níveis plasmáticos de AST entre os grupos do estudo não apresentou diferenças estatisticamente significativas. A análise isolada da meia-vida da AST em relação à ALT, respectivamente de 17 e 47 horas, pode explicar, em parte, os dados obtidos.

No presente estudo, os grupos que utilizaram álcool foram submetidos a duas semanas de abstinência, portanto a meia-vida curta de AST pode ter influenciado nos resultados. Torna-se importante considerar também que as aminotransferases não são enzimas específicas do consumo do álcool e outras condições clínicas podem alterá-las como as hepatopatias de diferentes etiologias, miopatias e miocardiopatias.

Este estudo demonstrou elevações na atividade plasmática da enzima ALP no grupo de animais que recebeu álcool e permaneceu sedentário (AS) durante protocolo experimental, quando comparado com o grupo que permaneceu sedentário sem ingerir álcool (CS) e com o grupo que recebeu álcool e exercitou. Tais resultados sugerem que nos dois grupos onde houve consumo de álcool houve menor injúria hepática naquele que praticou atividade física, em relação ao grupo sedentário.

A GGT tem alta sensibilidade no alcoolismo, sendo um dos principais marcadores utilizados no controle clínico de pacientes etilis$\operatorname{tas}^{30}$, no entanto seus valores não se mostraram alterados nos grupos durante o estudo. Apesar da correlação positiva e estatisticamente significativa $(r=0,898 ; p<0,001)$ observada entre os dois kits utilizados para a dosagem de GGT, os valores obtidos neste estudo foram extremamente baixos, não apresentando diferenças significativas entre os grupos estudados.
A correlação entre os níveis de GGT e o consumo de álcool é mais nítida entre os usuários crônicos ${ }^{30}$, e nenhum estudo foi encontrado na literatura quanto aos usuários de início recente. Um estudo com 30 etilistas crônicos do sexo masculino internados em um hospital psiquiátrico para desintoxicação e tratamento do alcoolismo identificou aumento nos níveis plasmáticos de GGT em 87\% dos pacientes ao ponto que os níveis de ALP estavam alterados em apenas $23 \%{ }^{28}$, discordando em ambos os parâmetros com o nosso estudo que demonstrou níveis inalterados de GGT em ratos consumidores de álcool e elevada atividade de ALP nos ratos do grupo AS quando comparado aos ratos de outros grupos.

Segundo Silva Junior et al. ${ }^{31}$, diferente do que ocorre em humanos, a GGT não é um marcador sensível para colestase extra-hepática em ratos Wistar ao passo que a ALP é a enzima ideal nessa situação. Diante de tais resultados acredita-se que possa haver diferenças no metabolismo hepático ou na distribuição dessas enzimas nas células canaliculares de ratos Wistar quando comparados aos seres humanos. Supõe-se ainda que os ratos que foram submetidos à atividade física e consumiram álcool apresentem menor risco de apresentar colestase extra-hepática se comparados aos ratos do grupo que ingeriu álcool e permaneceu sedentário.

No presente estudo, não foram observadas diferenças estatisticamente significativas nos níveis de amilase sérica entre os grupos. A amilase é uma enzima produzida no pâncreas e nas glândulas salivares e comumente encontra-se elevada nas pancreatites aguda e crônica de origem alcoólica ${ }^{32}$. No estudo anteriormente citado realizado com 30 pacientes em um hospital psiquiátrico observou-se hiperamilasemia em 20\% dos indivíduos etilistas ${ }^{33}$.

Não foi encontrado na literatura nenhum estudo que correlacionasse os níveis de amilase com o consumo de álcool em ratos submetidos a regime de atividade física regular. Porém, o curto período de ingestão de álcool pelos ratos do presente estudo pode justificar, em parte, os valores obtidos para a enzima amilase. Alcoólatras que apresentam tempo pequeno de consumo de álcool, considerado como dependência aguda, sofrem poucas alterações hepáticas devido à alta capacidade de regeneração do fígado ${ }^{29}$. Outra limitação observada neste estudo foi que o exercício físico realizado foi baseado em experimentos anteriores ${ }^{33}$, sendo o tempo inferior às oito semanas, descrito na literatura como tempo mínimo para o organismo se adaptar às alterações metabólicas e fisiológicas sofridas durante o tempo de treinamento. Todavia, caso o protocolo experimental de exercícios ultrapassasse duas semanas, a chance de regeneração hepática após o tratamento com álcool poderia prejudicar a análise das enzimas.

\section{CONCLUSÕES}

Os resultados do presente estudo permitem concluir que a associação entre consumo de álcool e sedentarismo aumentou a liberação das enzimas ALT e ALP em ratos Wistar; e a prática de exercício físico aeróbico após abstinência alcoólica evitou o aumento na liberação plasmática de ALP nestes animais.

\section{AGRADECIMENTOS}

AJ Natali é bolsista de produtividade do Conselho Nacional de Desenvolvimento Científico e Tecnológico (CNPq). LM Ribeiro é bolsista de iniciação científica do Conselho Nacional de Desenvolvimento Científico e Tecnológico (CNPq).

Todos os autores declararam não haver qualquer potencial conflito de interesses referente a este artigo. 
CONTRIBUIÇÕES DOS AUTORES: Cada autor contribuiu individual e significantemente para o desenvolvimento deste artigo. TR (0000-0003-2254-3084)*, LMR (00000002-9932-1749)* e LML (0000-0001-5349-1577)* foram os principais contribuintes na redação do manuscrito; TR, ACSP (0000-0001-8486-0531)*, CAC (0000-00019539-8653)* e DNQC (0000-0002-0354-7269) realizaram a parte experimental, acompanhando os tratamentos com os grupos e realizando a eutanásia; LML e CAC realizaram as análises laboratoriais e a análise estatista dos dados; LML, AJN (0000-0002-4927-4024)* e ETP (0000-0002-4927-4024)* realizaram a revisão do manuscrito e contribuíram com o conceito intelectual do estudo. *Número ORCID (Open Researcher and Contributor ID).

\section{REFERÊNCIAS}

1. Carlini EA. II Levantamento domiciliar sobre o uso de drogas psicotrópicas no Brasil: estudo envolvendo as 108 maiores cidades do país. São Paulo: CEBRID - Centro Brasileiro de Informação sobre Drogas Psicotrópicas: UNIFESP - Universidade Federal de São Paulo; 2006.

2. Sousa FSP, Oliveira EN. Caracterização das internações de dependentes químicos em Unidade de Internação Psiquiátrica do Hospital Geral. Ciênc Saúde Coletiva. 2010;15(3):671-7.

3. Zaleski M, Morato GS, Silva VA, Lemos T. Aspectos neurofarmacológicos do uso crônico e da Síndrome de Abstinência do Álcool. Rev Bras Psiquiatr. 2004; 26(Suppl.1):40-2.

4. Guimarães W, Florindo $A A$, Stopa $S R$, César CLG, Barros MBA, Carandina L, et al. Consumo abusivo e dependência de álcool em população adulta no Estado de São Paulo, Brasil. Rev Bras Epidemiol. 2010;13(2):314-25.

5. Xavier AM, Goessler KF, Ferrari O, Juliani LC, de Andrade FG, Ramos SDP. Efeito do treinamento físico no pulmão de ratos submetidos à ingestão alcoólica. Rev Bras Med do Esporte. 2010;16(6):436-40.

6. Paljärvi T, Mäkelä P, Poikolainen K. Pattern of drinking and fatal injury: a population-based follow-up study of Finnish men. Addiction. 2005;100(12):1851-9.

7. Salomé $H$, French $M$, Matzger $H$, Weisner $C$. Alcohol consumption, risk of injury, and high-cost medical care. J Behav Health Serv Res. 2005;32(4):368-80.

8. Polednak A. Recent trends in incidence rates for selected alcohol-related cancers in the United States. Alcohol Alcohol. 2005;40(3):234-8.

9. Cook R, Clark D. Is there an association between alcohol consumption and sexually transmitted diseases? A systematic review. Sex Transm Dis. 2005;32(3):156-64

10. Mathews S, Xu M, Wang H, Bertola A, Gao B. Animals models of gastrointestinal and liver diseases. Animal models of alcohol-induced liver disease: pathophysiology, translational relevance, and challenges. Am J Physiol Gastrointest Liver Physiol. 2014;306(10):G819-23.

11. Xie Y-D, Feng B, Gao Y, Wei L. Characteristics of alcoholic liver disease and predictive factors for mortality of patients with alcoholic cirrhosis. Hepatobiliary Pancreat Dis Int. 2013;12(6):594-601.

12. Liu J. Ethanol and liver: Recent insights into the mechanisms of ethanol-induced fatty liver. World Gastroenterol. 2014;20(40):14672-85.

13. Whitfield JB', Heath AC, Madden PA, Pergadia ML, Montgomery GW, Martin NG. Metabolic and biochemical effects of low-to-moderate alcohol consumption. Alcohol Clin Exp Res. 2013;37(4):575-86.

14. Castro LA, Baltieri DA. [The pharmacologic treatment of the alcohol dependence]. Rev Bras Psiquiatr. 2004;26(Suppl 1):S43-6.

15. Brager AJ, Hammer SB. Impact of wheel running on chronic ethanol intake in aged Syrian hamsters. Physiol Behav. 2012;107(3):418-23.

16. Mello MT, Boscolo RA, Esteves, AM, Tufik S. O exercício físico e os aspectos psicobiológicos. Rev Bras Med Esporte. 2005;11(3):203-7.

17. Wang Z, Su B, Fan S, Fei H, Zhao W. Protective effect of oligomeric proanthocyanidins against alcohol-induced liver steatosis and injury in mice. Biochem Biophys Res Commun. 2015:458(4):757-62.
18. Bell RL, Sable HJK, Colombo G, Hyytia P, Rodd ZA, Lumeng L. Animal models for medications development targeting alcohol abuse using selectively bred rat lines: neurobiological and pharmacologica validity. Pharmacol Biochem Behav. 2012;103(1):119-55.

19. Ardies CM, Morris GS, Erickson CK, Farrar RP. Both acute and chronic exercise enhance in vivo ethano clearance in rats. J Appl Physiol. 1985. 1989;66(2):555-60

20. Mallikarjuna K, Shanmugam KR, Nishanth K, Wu MC, Hou CW, Kuo CH, et al. Alcohol-induced deterioration in primary antioxidant and glutathione family enzyme reversed by exercice training in the liver of old rats. Alcohol. 2010;44(6):523-9.

21. Mallikarjuna K, Nishanth K, Hou CW, Kuo CH, Sathyavelu RK. Effect of exercise training on ethanolinduced oxidative damage in aged rats. Alcohol. 2009;43(1):59-64.

22. Adams LA, Knuiman MW, Divitini ML, Olynyk JK. Body mass index is a stronger predictor of alanine aminotransaminase levels than alcohol consumption. J Gastroenterol Hepatol. 2008;23(7 Pt 1):1089-93.

23. Lee $\mathrm{DH}, \mathrm{Ha} \mathrm{MH}$, Christiani DC. Body weight, alcohol consumption and liver enzyme activity - a 4-year follow-up study. Int J Epidemiol. 2001;30(4):766-70.

24. Conigrave KM, Davies P, Haber P, Whitfield JB. Traditional markers of excessive alcohol use. Addiction. 2003;98(Suppl 2):31-43.

25. Das SK, Vasudevan DM. Effect of ethanol on liver antioxidant defense systems: a dose dependent study. Ind J Clin Biochem. 2005;20(1):80-4

26. Ardies CM, Morris GS, Erickson CK, Farrar RP. Effects of exercise ans ethanol on liver mitochondrial function. Life Sci. 1987;40(11):1053-61.

27. Indira, SK, Jhansi LC. Endurance exercise-induced alterations in antioxidant enzymes of old albino male rats. Curr Sci. 2001;80:921-3.

28. Gunduz F, Senturk UK, Kuru O, Aktekin B, Aktekin MR. The effect of one year's swimming exercise on oxidant stress and antioxidant capacity in aged rats. Physiol Res. 2004;53(2):171-6.

29. Ying WC, Lin HL, Chen CW, Lin JN, Kuo LC, Cheng YC, et al. Relationship Between Blood Alcoho Concentration and Hepatic Enzymes in an Emergency Department. Tzu Chi Med J. 2010;22(1):24-8.

30. Deguti MM, Goncalves LL. Marcadores biológicos dos alcoolismo. Rev Psiquiatr Clin. 2000;27(1):5-9.

31. Silva Junior OC, Coelho AFB, Souza MEJ, Picinato MANC, Franco CFF, Vanni JC. Sensitivity of alkaline phosphatase and gama-glutamyl transferase as markers of biliary extrahepatic obstruction in rats. Medicina (FMRP). 1993; 26(1):41-4.

32. Pacheco RC, Oliveira LCM. Relação lipase/amilase nas pancreatites agudas de causa biliar e nas pancreatites agudas/crônicas agudizadas de causa alcoólica. Arq Gastroenterol. 2007:44(1):35-8.

33. Borini P, Guimaraes RC. Indicators of inflammation and cellular damage in chronic asymptomatic or oligosymptomatic alcoholics: correlation with alteration of bilirubin and hepatic and pancreatic enzymes. Rev Hosp Clin Fac Med Sao Paulo. 1999:54(2):53-60. 